Hope College

Hope College Digital Commons

Faculty Publications

2001

\title{
Granting Forgiveness or Harboring Grudges: Implications for Emotion, Physiology, and Health
}

Charlotte vanOyen-Witvliet

Hope College,witvliet@hope.edu

Thomas Ludwig

Hope College, ludwig@hope.edu

Kelly L. Vander Laan

Hope College

Follow this and additional works at: https://digitalcommons.hope.edu/faculty_publications

Part of the Psychology Commons

\section{Recommended Citation}

Witvliet, Charlotte van Oyen, Thomas E. Ludwig, and Kelly L. Vander Laan. "Granting Forgiveness or Harboring Grudges: Implications for Emotion, Physiology, and Health." Psychological Science 12, no. 2 (March 1, 2001): 117-23. doi:10.1111/1467-9280.00320.

This Article is brought to you for free and open access by Hope College Digital Commons. It has been accepted for inclusion in Faculty Publications by an authorized administrator of Hope College Digital Commons. For more information, please contact digitalcommons@hope.edu. 
Running Head: Granting Forgiveness or Harboring Grudges

\title{
Citation:
}

Witvliet, C.V.O., Ludwig, T.E., \& Vander Laan, K.L. (2001). Granting forgiveness or harboring grudges: Implications for emotion, physiology, and health. Psychological Science,121, 117-123.

\author{
Granting Forgiveness or Harboring Grudges:
}

Implications for Emotion, Physiology, and Health

Charlotte vanOyen Witvliet, Thomas E. Ludwig, \& Kelly L. Vander Laan

Hope College

Correspondence may be directed to Charlotte vanOyen Witvliet, Ph.D., Psychology Department, Hope College, Holland, MI 49422-9000; email: witvliet@hope.edu.

This research was supported by a grant to Charlotte vanOyen Witvliet from the John Templeton Foundation for Scientific Studies on the Subject of Forgiveness. We wish to thank Erin Thompson, Dennis Ahmad, Jenette Bongiorno, January Estes, Emily Hollebeek, Daniel Kubacki, Michelle Lynch, Renata Meixner, Sharon Schultz, Sarah Snyder, and Dara Spearman for assistance with data collection. 


\begin{abstract}
Interpersonal offenses frequently mar relationships. Theorists have argued that the responses victims adopt toward their offenders have ramifications not only for their cognition, but also for their emotion, physiology, and health. This study examined the immediate emotional and physiological effects that occurred when participants (35 females, 36 males) rehearsed hurtful memories and nursed grudges (i.e., were unforgiving) compared to when they cultivated empathic perspective-taking and imagined granting forgiveness (i.e., were forgiving) toward real-life offenders. Unforgiving thoughts prompted more aversive emotion, and significantly higher corrugator (brow) EMG, skin conductance, heart rate, and blood pressure change scores from baseline. These EMG, skin conductance, and heart rate effects persisted after imagery into the recovery periods. Forgiving thoughts prompted greater perceived control and comparatively lower physiological stress responses. Results dovetail with the psychophysiology literature and suggest possible mechanisms through which chronic unforgiving responses may erode health while forgiving responses may enhance it.
\end{abstract}


Granting Forgiveness or Harboring Grudges: Implications for Emotion, Physiology, and Health

Social relationships are often marred by interpersonal offenses. An expanding group of theorists, therapists, and health professionals has proposed that the ways we respond to interpersonal offenses can significantly affect our health (McCullough, Sandage, \& Worthington, 1997; McCullough \& Worthington, 1994; Thoresen, Harris, \& Luskin, 1999). Unforgiving responses (rehearsing the hurt, harboring a grudge) are considered health-eroding, while forgiving responses (empathizing with the human condition of the offender, granting forgiveness) are thought to be health-enhancing (e.g., Thoresen et al., 1999; Williams \& Williams, 1993). Whereas several published studies have found a positive relationship between forgiveness and mental health variables (Al-Mabuk, Enright, \& Cardis, 1995; Coyle \& Enright, 1997; Freedman \& Enright, 1996; Hebl \& Enright, 1993), the current literature lacks controlled studies of forgiveness and physical health-related variables.

Yet, indirect evidence suggests that the health implications of forgiveness and unforgiveness may be substantial. Research associates the unforgiving responses of blame, anger, and hostility with impaired health (Affleck, Tennen, Croog, \& Levine, 1987; Tennen \& Affleck, 1990), particularly coronary heart disease and premature death (Miller, Smith, Turner, Guijarro, \& Hallet, 1996). Further, research suggests that reductions in hostility—brought about by behavioral interventions that emphasize becoming forgiving — are associated with reductions in coronary problems (Friedman et al., 1986; Kaplan, 1992).

Another line of research suggests that granting or withholding forgiveness may influence cardiovascular health through changes in allostasis and allostatic load. Allostasis involves changes in the multiple physiological systems that allow people to survive the demands of both internal and external stressors (McEwen, 1998). Although allostasis is necessary for survival, extended physiological stress responses triggered by psychosocial factors such as anxiety and hostility can result in allostatic load, eventually leading to physical breakdown. Interpersonal transgressions and people's adverse reactions to them may contribute to allostatic load and health risk through sympathetic nervous system, endocrine, and immune system changes (e.g., KiecoltGlaser, 1999). In contrast, forgiveness may buffer health by reducing physiological reactivity and allostatic load (Thoresen et al., 1999). 


\section{A Theoretical Framework}

An understanding of the relationships among unforgiving responses, forgiving responses, physiology, emotion, and health may benefit from the established framework of bioinformational theory (Lang, 1979, 1995). Lang posits that physiological responses are essential aspects of emotional experiences, memories, and imagined responses. An extensive literature has supported this view, documenting that physiological responses reliably vary depending on the emotional experiences people think about, or imagine (e.g., Cook, Hawk, Davis, \& Stevenson, 1991; Lang, 1979; Witvliet \& Vrana, 1995, 2000). Two emotional dimensions strongly influence the physiological reactions that occur: valence (negative - positive) and arousal (e.g., Lang, 1995; Witvliet \& Vrana, 1995). For example, the valence of emotion is important for facial expressions, with negative imagery stimulating greater muscle tension in the brow (Witvliet \& Vrana, 1995). With heightened emotional arousal, cardiovascular measures such as blood pressure (e.g., Yogo, Hama, Yogo, \& Matsuyama, 1995) and heart rate show greater reactivity, and skin conductance —an index of sympathetic nervous system activity—is also more reactive (e.g., Witvliet \& Vrana, 1995).

Interpersonal transgressions are emotionally laden experiences that often stimulate negative and arousing memories or imagined emotional responses (e.g., grudges). Within Lang's theory, we would predict that unforgiving memories and mental imagery would produce negative facial expressions and increased cardiovascular and sympathetic reactivity, similar to other negative and arousing emotions (e.g., fear, anger). In contrast, forgiving responses should reduce the negativity and intensity of a victim’s emotional response, quelling these physiological reactions, as more pleasant and relaxing imagery does (Witvliet \& Vrana, 1995). In terms of allostasis (McEwen, 1998), emotional states (e.g., unforgiving responses) that intensify and extend cardiovascular and sympathetic reactivity would increase allostatic load, whereas those that limit these physiological reactions (e.g., forgiving responses) would improve health.

\section{Particular Unforgiving and Forgiving Responses to Interpersonal Transgressions}

The forgiveness literature has focused on the effects of two unforgiving responses (rehearsing the hurt, harboring a grudge) and two forgiving responses (developing empathy for the offender's humanity, granting forgiveness) to interpersonal violations. 


\section{$\underline{\text { Unforgiving Responses }}$}

Rehearsing the hurt. Once hurt, people often rehearse memories of the painful experience, even unintentionally, perhaps because the physiological reactivity that occurs during emotionally significant events facilitates memory encoding and retrieval (cf. Witvliet, 1997). When people rehearse hurtful memories, they may perpetuate negative emotion and adverse physiological effects (Witvliet, 1997; Worthington, 1998). Interestingly, Huang and Enright (2000) found that in the first minute of describing a past experience with conflict (versus describing a typical day), those who had forgiven due to religious pressure showed greater blood pressure increases compared to those who had forgiven because of unconditional love.

Harboring a grudge. When people hold a grudge, they stay in the victim role and perpetuate negative emotions associated with rehearsing the hurtful offense (Baumeister, Exline, \& Sommer, 1998). Despite this, victims may be drawn to hold grudges because they may secure tangible or emotional benefits, such as a regained sense of control or a sense of "saving face" (Baumeister et al., 1998). Yet, nursing a grudge is considered "a commitment to remain angry (or to resume anger periodically)," and to endure the adverse health effects associated with anger and blame (Baumeister et al., 1998, p. 98).

\section{Forgiving Responses}

Developing feelings of empathy for the perpetrator is considered to play a pivotal role in turning the victim away from unforgiveness and beginning the forgiveness process (Worthington, 1998). Empathy involves thinking of the offender's humanity (rather than defining the person solely in terms of the offense) and trying to understand what factors may have influenced the offending behavior (Enright \& Coyle, 1998). When victims engage in this sort of perspective-taking, the resulting empathic compassion reduces the intense arousal and negative valence of hurts and grudges and introduces more positively valent emotion for the victim (McCullough et al., 1997). Empathy is also thought to shift victims' facial expressions and reduce their cardiovascular and sympathetic nervous system stress responses (Worthington, 1998).

Granting forgiveness builds on the core of empathy and involves cognitive, emotional, and possibly behavioral responses (McCullough et al., 1997). It is important to note that forgiveness still allows for holding the offender responsible for what was done, and does not involve denying, ignoring, minimizing, tolerating, condoning, excusing, or forgetting the offense (see Enright \& Coyle, 1998). Although no universal definition of 
forgiveness exists, forgiveness theorists emphasize that it involves letting go of the negative feelings and adopting a merciful attitude of goodwill toward the offender (Thoresen, Luskin, \& Harris, 1998). This may free the wounded person from a prison of hurt and vengeful emotion, yielding both emotional and physical benefits, including reduced stress, less negative emotion, fewer cardiovascular problems, and improved immune system performance (McCullough et al., 1997; Worthington, 1998).

Applying the Emotional Imagery Paradigm

Unforgiving responses (rehearsing the hurt, harboring a grudge) may erode health by activating negative, intense emotion and cardiovascular and sympathetic nervous system reactivity. Forgiving responses (empathizing with the offender’s humanity, granting forgiveness) may buffer health or promote healing by quelling cardiovascular reactivity and sympathetic nervous system hyperarousal (Thoresen et al., 1999). In this study, we investigated these hypotheses by measuring physiology continuously as each participant thought about a real-life offender in unforgiving and forgiving ways, providing a window into the moment-by-moment effects of choosing each response. We used a within-subjects repeated measures design (Vrana \& Lang, 1990; Witvliet \& Vrana, 1995, 2000), allowing us to compare the different physical effects of adopting unforgiving versus forgiving responses to a particular offender. Building on the psychophysiology literature relevant to health, we measured imagery effects on self-reports of emotion valence and emotional arousal, self-reports of perceived control, anger, and sadness, facial EMG measured at the corrugator (brow) region ${ }^{1}$, skin conductance (as an indicator of sympathetic nervous system activity), heart rate, and blood pressure. We hypothesized that unforgiving imagery would prompt more negative and arousing emotion and higher perceived control. We also predicted greater increases in corrugator muscle tension associated with negative emotion, and higher skin conductance, heart rate and blood pressure change scores associated with heightened emotional arousal during unforgiving imagery.

Given the importance that extended physiological reactivity may have for allostatic load and health consequences (e.g., McEwen, 1998), we examined whether differences between unforgiving and forgiving effects would persist after imagery periods, when participants tried to stop their imagery and engaged in a relaxation task. Although not previously tested, evidence from the trauma literature suggests that negative and arousing personal imagery that evokes heightened physiological reactivity is difficult to quell (cf. Witvliet, 
1997). Physiological differences may also persist because the valence and arousal of unforgiving imagery differs considerably from the target mood of relaxation. If so, unforgiving responses to interpersonal offenses may contribute to more adverse health effects because the heightened cardiovascular and sympathetic nervous system reactivity both during and after imagery may increase allostatic load.

\section{Method}

This study used a standard within-subjects emotional imagery paradigm (Vrana \& Lang, 1990; Witvliet \& Vrana, 1995, 2000), adapting it to study the emotional and physiological effects of imagining unforgiving and forgiving responses to an interpersonal offender.

\section{$\underline{\text { Participants }}$}

Seventy-two introductory psychology students voluntarily participated in this experiment. Because one female discontinued the study before its conclusion, the data for seventy-one (36 male, 35 female) participants are reported. Data for two participants were excluded from blood pressure analyses because of equipment problems.

\section{$\underline{\text { Stimulus Materials }}$}

The imagery script materials used to prompt autobiographical forgiveness-related imagery were based on the forgiveness literature (McCullough et al., 1997). All participants used the same unforgiving scripts (rehearsing the hurt, harboring a grudge) and forgiving scripts (empathizing with the offender, granting forgiveness) to maximize internal validity. To maximize external validity, all of the unforgiving and forgiving scripts instructed each participant to apply the unforgiving and forgiving responses to a single interpersonal offense from his or her life. This approach allowed us to assess the emotional and physiological effects choosing to adopt unforgiving versus forgiving responses to a particular real life offender. All imagery scripts encouraged each participant to consider the thoughts, feelings, and physical responses that would accompany each type of unforgiving and forgiving response.

\section{$\underline{\text { Apparatus }}$}

We used a Dell 486 computer to time the experimental events and collect data using on-line physiological data collection software (VPM; Cook, Atkinson, \& Lang, 1987). Auditory tones at three frequencies—-high 
$(1350 \mathrm{~Hz})$, medium $(985 \mathrm{~Hz})$, and low $(620 \mathrm{~Hz})$ — signaled imagery and relaxation trials. The tones were 500 ms long and $73 \mathrm{~dB}[\mathrm{~A}]$. They were generated by a Coulbourn V85-05 Audio Source Module voltage controlled oscillator with a selectable envelope rise/fall gate set for an 80-ms rise/fall time. The tones were presented to the participant through both headphones and speakers.

Facial EMG was recorded at the corrugator (i.e., brow) muscle region ${ }^{1}$ using sensor placements suggested by Fridlund \& Cacioppo (1986). Facial skin was prepared using an alcohol pad and Medical Associates electrode gel. Then miniature Ag-AgCl electrodes filled with Medical Associates electrode gel were applied. EMG signals were amplified (x 50,000) by a Hi Gain V75-01 bioamplifier, using 90-Hz high-pass and 1-kHz low-pass filters. A Coulbourn multi-function V76-23 integrator (nominal time constant $=10 \mathrm{~ms}$ ) then rectified and integrated the signals.

Skin conductance levels were measured by a Coulbourn isolated skin conductance V71-23 coupler using an applied constant voltage of $0.5 \mathrm{~V}$ across two standard electrodes. Electrodes were filled with a mixture of physiological saline and Unibase (Fowles, Christie, Edelberg, Grings, Lykken, \& Venables, 1981) and applied to the hypothenar eminence on the left hand after it was rinsed with tap water. A 12-bit analog-digital converter sampled the skin conductance and facial EMG channels at $10 \mathrm{~Hz}$.

Electrocardiogram data were collected using two standard electrodes, one on each forearm. A Hi Gain V75-01 bioamplifier amplified and filtered the signals. The signals were then sent to a digital input on the computer that detected R-waves, and measured interbeat intervals in milliseconds.

We continuously measured blood pressure at each heartbeat with an Ohmeda 2300 Non-Invasive Blood Pressure Monitor, placing the cuff between the first and second knuckles on the middle finger of the left hand. ${ }^{2}$ Procedure

Each participant completed a two-part, two-hour testing session. First, the participant identified a particular person he or she blamed for mistreating, offending, or hurting him or her. Then the participant completed a questionnaire about the nature of the offense and his or her responses to it (see footnote 5). Second, in the imagery phase of the study, the participant actively imagined each type of unforgiving and forgiving response to the previously identified offender eight times in systematically manipulated orders that were counterbalanced across participants. The study session was divided into blocks of trials, with two types of imagery trials in each 
block. Acoustic tones (high, low) were used to signal exactly when the participant was to imagine a specific type of forgiving or unforgiving response. Medium tones signaled participants to engage in a relaxation task, thinking the word “one” every time they exhaled (e.g., Vrana \& Lang, 1990; Witvliet \& Vrana, 1995, 2000).

During imagery, physiology was monitored continuously during trials consisting of an 8-s baseline (relaxation) period, 16-s imagery period, and 8-s recovery (relaxation) period. On-line monitoring allowed us to measure the immediate psychophysiological effects of people's unforgiving and forgiving responses as they occurred.

After each block of imagery trials, participants rated their feelings during the preceding two types of imagery. Using a video display and computer joystick (see Hodes, Cook, \& Lang, 1985), participants rated their level of emotional valence (negative - positive) and arousal (low - high), as well as anger, sadness, and perceived control. As a manipulation check, participants also rated how much empathy they felt for the offender and how much they felt they had forgiven the offender during the different imagery conditions ("not at all”“completely”). All ratings were converted to a scale ranging from 0 to 20. Participants privately registered all ratings directly into a computer and were encouraged to be completely honest.

\section{Data Collection and Reduction}

During the experiment, the participant's heart rate and blood pressure were measured on a heartbeat-toheartbeat basis, and their facial EMG and skin conductance level data were measured on a second-to-second basis. Cardiac interbeat intervals were converted off-line to heart rate in beats per minute for each imagery period. Within each type of imagery condition (hurt, grudge, empathy, forgiveness) the physiology measures were averaged over 4-s epochs, resulting in two 4-s epochs during the baseline period, four 4-s epochs during the imagery period, and two 4-s epochs during the recovery period. During the imagery and recovery periods, change scores for each 4-s epoch were created by subtracting data values from the 4-s baseline epoch immediately before the imagery period.

For the analyses, data for the hurt and grudge imagery trials were averaged to form an unforgiving condition because these two conditions are emotionally negative and arousing and are often experienced together (see Baumeister et al., 1998). Data for the empathy and forgiveness imagery trials were averaged to form a forgiving condition because these two conditions are more positive and less arousing, and empathy is 
considered central to the forgiveness process (Worthington, 1998). The averaged data in the unforgiving conditions were compared to the averaged data in the forgiving conditions using analyses of variance (ANOVAs) with repeated measures. ${ }^{3}$ During imagery and recovery periods, the overall effect of emotion condition (forgiving vs. unforgiving imagery) was assessed. ${ }^{4}$

Results

\section{$\underline{\text { Self-Reports }}$}

Interpersonal offenses. Participants reported that their primary offenders included friends, romantic partners, parents, and siblings. Common offenses included betrayals of trust, rejection, lies, and insults. ${ }^{5}$

Ratings. Ratings results were consistent with predictions (Table 1). During unforgiving imagery, participants rated feeling more negatively valent $[\underline{F}(1,70)=203.46, \underline{\mathrm{p}}<.001]$, aroused $[\underline{\mathrm{F}}(1,70)=307.24, \underline{\mathrm{p}}<$ $.001]$, angry $[\underline{F}(1,70)=466.56, \underline{\mathrm{p}}<.001]$, sad $[\underline{F}(1,70)=55.48, \underline{\mathrm{p}}<.001]$, and less in-control $[\underline{F}(1,70)=81.02$, $\underline{\mathrm{p}}<.001$ ]. During the forgiving imagery, participants reported significantly greater empathy for, $\underline{\mathrm{F}}(1,70)=$ 326.74, $\mathrm{p}<.001$, and forgiveness toward the offender, $\underline{\mathrm{F}}(1,70)=353.87, \mathrm{p}<.001$.

\section{Corrugator EMG}

Figure 1 shows that corrugator EMG change scores were significantly higher both during the unforgiving imagery, $\underline{\mathrm{F}}(1,70)=14.43, \underline{\mathrm{p}}<.001$, and the unforgiving recovery period, $\underline{\mathrm{F}}(1,70)=13.79, \underline{\mathrm{p}}<.001$. These predicted findings parallel the strong relationship between corrugator EMG and negative valence in the literature (see Fridlund \& Izard, 1983; Witvliet \& Vrana, 1995). The recovery period data further suggest that negative emotion persisted despite efforts to "turn off" the imagery and relax.

\section{$\underline{\text { Skin Conductance Levels }}$}

As depicted in Figure 2, tonic skin conductance levels (SCLs) showed a general decrease both during and after imagery, a pattern reflecting habituation to the experimental context.

Importantly, skin conductance level (SCL) change scores were significantly lower during the forgiving imagery conditions, $\underline{\mathrm{F}}(1,70)=14.58, \underline{\mathrm{p}}<.001$, and during forgiving recovery periods, $\underline{\mathrm{F}}(1,70)=18.62, \underline{\mathrm{p}}<$ .001, indicating comparatively less SNS arousal. Conversely, SCLs resisted habituation and were higher during the unforgiving imagery and recovery periods, as hypothesized. This pattern dovetails with participant reports 
of higher arousal during the unforgiving conditions. This result is striking because emotional differences must be highly potent to yield significant effects on SCLs in imagery paradigms (Witvliet \& Vrana, 1995), and here the differences in SNS activation persisted even as participants tried to quell their responses and relax.

\section{$\underline{\text { Heart Rate }}$}

As depicted in Figure 3, heart rate (HR) increased from baseline regardless of how participants imagined responding to their offenders, a pattern found in other studies of personalized emotional imagery (Witvliet \& Vrana, 1995, 2000).

As hypothesized, HR increases were greater during unforgiving imagery, $\underline{\mathrm{F}}(1,70)=34.94, \underline{\mathrm{p}}<.001$, and during unforgiving recovery periods, $\underline{\mathrm{F}}(1,70)=14.46, \mathrm{p}<.001$, similar to the persisting SCL and corrugator EMG effects. This finding is consistent with the arousal ratings, SCL data, and findings in the literature, in which significantly greater HR increases occurred during highly arousing imagery (e.g., Cook et al., 1991; Witvliet \& Vrana, 1995, 2000). Together with the corrugator and SCL results, these data suggest that it is difficult to quell the aversive emotion and the concomitant physiological reactivity of unforgiving imagery.

\section{$\underline{\text { Mean Arterial Pressure }}$}

Figure 4 shows that mean arterial pressure (MAP) increases were significantly greater during the unforgiving than the forgiving conditions, $\underline{\mathrm{F}}(1,68)=8.98, \underline{\mathrm{p}}<.01$, as hypothesized. This finding parallels the HR data, ratings results, and findings in the literature, which link blood pressure reactivity to higher levels of arousal (e.g., Yogo et al., 1995) and anger (e.g., Kunzendorf, Cohen, Francis, \& Cutler, 1996). During the recovery periods, MAP did not differ significantly between conditions, $\underline{\mathrm{F}}(1,68)=.185, \underline{\mathrm{p}}=.668$.

\section{Discussion}

The physiology of forgiveness and unforgiveness is uncharted territory for empirical study, despite theoretical explorations of the possible health costs of unforgiveness and health benefits of forgiveness (e.g., McCullough et al., 1997; Williams \& Williams, 1993). In this study, we investigated the emotional and physiological effects that occurred when people imagined responding to their real-life offenders in unforgiving ways (rehearsing the hurt, harboring a grudge) and forgiving ways (empathic perspective-taking, granting forgiveness). 
Consistent with bioinformational theory (Lang, 1979, 1995), imagery of unforgiving and forgiving responses to a particular offender yielded differences in both self-reported emotion and physiological responding. Participants felt significantly more negative, aroused, angry, sad, and less in-control during the unforgiving conditions (Table 1). Participants also showed greater facial tension at the corrugator (brow) muscle region during unforgiving imagery (Figure 1), paralleling negative emotion effects in the literature (see Fridlund \& Izard, 1983; Witvliet \& Vrana, 1995). During the arousing unforgiving imagery, participants experienced significantly greater sympathetic nervous system (SNS) arousal—as indicated by higher skin conductance level change scores (Figure 2)—and greater cardiovascular reactivity in terms of heart rate and blood pressure (Figures 3 and 4). These results parallel arousal effects in the literature (e.g., Witvliet \& Vrana, 1995; Yogo et al., 1995). Further, the elevated corrugator EMG, skin conductance, and heart rate change scores during unforgiving imagery persisted into the post-imagery relaxing recovery periods. Overall, the physiological patterns in this study are quite consistent with the patterns that occur during emotional imagery in general (Witvliet \& Vrana, 1995), suggesting that the physiological effects of unforgiving and forgiving responses to interpersonal offenses may be influenced substantially by the emotional quality of these responses.

\section{$\underline{\text { Health Implications }}$}

These four physiological measures give us a window into what happens to our bodies during emotional thoughts about an offender, even when the thoughts are very brief. While it is unlikely that the brief unforgiving trials in this study would have a clinically significant effect on health, we believe that the effects obtained in this study provide a conservative measure of effects that naturally occur during unforgiving responses to real-life offenders. Lang (1979) has argued that physiological effects during emotional imagery mirror naturally occurring effects, but are less potent. In daily life, people may intensify their hurtful memories and vengeful thoughts (e.g., embellishing accounts of the offense with language that heightens contempt) and punctuate their imagery with overt behaviors (e.g., slamming doors, shouting), that intensify and extend blood pressure surges, heart rate elevations, and sympathetic nervous system (SNS) activation.

The emotional and physiological effects identified in this study may be mediators of a relationship between forgiveness and health (Thoresen et al., 1999). Earlier work identifies anger, hostility, anxiety, and depression as psychosocial risk factors for heart disease, and chronic SNS arousal as a mechanism for the 
relationship between psychosocial factors and heart disease (Allen \& Scheidt, 1996). This pattern is reflected in the current study, as participants reported significantly higher anger and sadness, and lower perceived control during unforgiving imagery, as well as greater SNS arousal and cardiovascular reactivity during unforgiving imagery.

Chronic unforgiving, begrudging responses may contribute to adverse health outcomes by perpetuating anger and heightening SNS arousal and cardiovascular reactivity. Anger expression has been strongly associated with chronically elevated blood pressure (Schwenkmezger \& Hank, 1996) and with the aggregation of platelets, which may increase vulnerability for heart disease (Wenneberg et al., 1997), especially if expressions of anger are frequent and enduring (see Thoresen et al., 1999). While fleeting feelings of unforgiveness may not be health-eroding, more frequent, intense, and sustained unforgiving emotional imagery and behaviors may create physiological vulnerabilities or exacerbate existing problems in a way that erodes health.

SNS arousal may also influence immune system functioning (Kiecolt-Glaser, Malarkey, Cacioppo, \& Glaser, 1994; Thoresen et al., 1999). For example, research suggests that marital discord can induce changes in SNS, endocrine, and immune system functioning, even in those reporting high marital satisfaction and living healthy lifestyles (Kiecolt-Glaser, 1999). When psychosocial stress is chronic, it may have the most impact on these physiological functions, thereby influencing susceptibility to and the progression of diseases (e.g., cancer, infectious illnesses). Conversely, interventions that buffer against psychosocial stressors, including interpersonal conflict, may ultimately influence health (see Kiecolt-Glaser \& Glaser, 1995).

The concept of allostasis (McEwen \& Stellar, 1993) may have considerable utility for understanding possible forgiveness-health links (Thoresen et al., 1999). Allostatic load can occur when physiological systems remain activated, despite termination of an external stressor (McEwen, 1998). In the present study, varied physiological responses (e.g., SCL, HR, BP, and facial EMG) were activated when people thought about responding to their offenders. This reactivity was significantly greater during unforgiving than forgiving imagery. Further, physiological reactivity remained significantly higher for SCLs, HR, and corrugator EMG even in the recovery period after imagery. This suggests that if unforgiving emotion is sufficiently potent and 
enduring, and if some physiological systems (e.g., SNS, cardiovascular) resist recovery, unforgiving responses could contribute to allostatic load.

In contrast, during the forgiving imagery, less HR, BP, and EMG reactivity occurred, and SCLs showed greater habituation. It may be that when people enact forgiving responses, the physiological demands of unforgiving emotional hurt and anger are reduced, thereby decreasing allostatic load and associated health risks. Interestingly, McEwen (1998) has advocated for the use of behavioral interventions that reduce stress, facilitate social support, and increase perceived control to improve allostasis and decrease allostatic load. Interventions to promote forgiveness have already begun to suggest an association between forgiveness and mental health (e.g., Al-Mabuk et al., 1995; Coyle \& Enright, 1997; Freedman \& Enright, 1996; Hebl \& Enright, 1993).

Furthermore, "increased frequency of forgiving others...could function to reduce the chronicity of distress (e.g., anger, blame, and vengeful thoughts and feelings) that has prospectively been shown to alter brain, coronary, and immune functioning. Such reductions could encourage diminished SNS arousal in frequency, magnitude and duration, resulting over time in less physical disease risk” (Thoresen et al., 1999; p. 259). This study begins to build the empirical case for this assertion.

Forgiveness research is still in its early development. We believe that this study - the first to explore the physiological effects of adopting various unforgiving and forgiving responses to real-life offenders_-provides a good foundation for future research. While people cannot undo past offenses, this study suggests that if people develop patterns of thinking about their offenders in forgiving ways rather than unforgiving ways, they may be able to change their emotions, physiological responses, and the health implications of a past they cannot change. 
References

Affleck, G., Tennen, H., Croog, S., \& Levine, S. (1987, Feb.). Causal attribution, perceived benefits, and morbidity after a heart attack: An 8-year study. Journal of Consulting \& Clinical Psychology, 55, 29-35.

Allan, R., \& Scheidt, S. (1996). Empirical basis for cardiac psychology. In R. Allan and S. Scheidt (Eds.) Heart and mind. (pp. 63-124). Washington, DC: American Psychological Association.

Al-Mabuk, R. H., Enright, R. D., \& Cardis, P. A. (1995). Forgiveness education with parentally lovedeprived late adolescents. Journal of Moral Education, 24, 427-444.

Baumeister, R. F., Exline, J. J., \& Sommer, K. L. (1998). The victim role, grudge theory, and two dimensions of forgiveness. In E.L. Worthington, Jr. (Ed.) Dimensions of forgiveness (pp. 79-104). Philadelphia, PA: Templeton Foundation Press.

Cook, E. W., III, Atkinson, L., \& Lang, K. G. (1987). Stimulus control and data acquisition for IBM PC's and compatibles. Psychophysiology, 24, 726-727.

Cook E. W., III, Hawk, L. W., Davis, T. L., \& Stevenson, V. E. (1991). Affective individual differences and startle reflex modulation. Journal of Abnormal Psychology, 24, 726-727.

Coyle, C. T., \& Enright, R. D. (1997). Forgiveness intervention with postabortion men. Journal Consulting and Clinical Psychology, 65, 1042-1046.

Enright, R. D., \& Coyle, C. T. (1998). Researching the process model of forgiveness within psychological interventions. In E. L. Worthington, Jr. (Ed.) Dimensions of forgiveness (pp. 139-161). Philadelphia, PA: Templeton Foundation Press.

Fowles, D. C., Christie, M. J., Edelberg, R., Grings, W. W., Lykken, D. T., \& Venables, P. H. (1981). Publication recommendations for electrodermal measurement. Psychophysiology, 18, 232-239.

Freedman, S. R., \& Enright, R. D. (1996). Forgiveness as an intervention goal with incest survivors. Journal of Consulting and Clinical Psychology, 64, 983-992.

Fridlund, A. J., \& Cacioppo, J. T. (1986). Guidelines for human electromyographic research. Psychophysiology, 23, 567-589. 
Fridlund, A. J., \& Izard, C. E., (1983). Electromyographic studies of facial expressions of emotions and patterns of emotions. In J. T. Cacioppo \& R. E. Petty (Eds.), Social psychophysiology: A sourcebook (pp. 243286). New York: Guilford.

Friedman, M., Thoresen, C., Gill, J., Ulmer, D., Powell, L. H., Price, V. A., Brown, B., Thompson, L., Rabin, D., Breall, W. S., Bourg, W., Levy, R., \& Dixon, T. (1986). Alterations of Type A behavior and its effects on cardiac recurrence in postmyocardial infarction patients: Summary results of the coronary prevention recurrence project. American Heart Journal, 112, 653-665.

Hebl, J. H., \& Enright, R. D. (1993). Forgiveness as a psychotherapeutic goal with elderly females. Psychotherapy, 30, 658-667.

Hodes, R. L., Cook, E. W., \& Lang, P. J. (1985). Individual differences in autonomic response: Conditioned association or conditioned fear? Psychophysiology, 22, 545-560.

Huang, S-T. T., \& Enright, R. D. (2000). Forgiveness and anger-related emotions in Taiwan: Implications for therapy. Psychotherapy, 37, 71-79.

Kaplan, B. (1992). Social health and the forgiving heart: the Type B story. Journal of Behavioral Medicine, 15, 3-14.

Kiecolt-Glaser, J. K. (1999). Stress, personal relationships, and immune function: Health implications. Brain, Behavior, and Immunity, 13, 61-72.

Kiecolt-Glaser, J. K., Glaser, R. (1995). Psychoneuroimmunology and health consequences: Data and shared mechanisms. Psychosomatic Medicine, 57, 269-274.

Kiecolt-Glaser, J. K., Malarkey, W. B., Cacioppo, J. T., \& Glaser, R. (1994). Stressful personal relationships: Immune and endocrine function. In R. Glaser and J. K. Glaser (Eds.) Handbook of human stress and immunity. (pp. 321-339). San Diego, CA: Academic Press, Inc.

Kunzendorf, R. G., Cohen, R., Francis, L., \& Cutler, J. (1996). Effect of negative imaging on heart rate and blood pressure, as a function of image vividness and image "realness.” Imagination, Cognition \& Personality, 16, 139-159.

Lang, P. J. (1995). The emotion probe: Studies of motivation and attention. American Psychologist, 50, 372-385. 
Lang, P. J. (1979). A bio-informational theory of emotional imagery. Psychophysiology, 16, 495-512.

McCullough, M. E., Sandage, S. J., \& Worthington, Jr., J. L. (1997). To forgive is human: How to put your past in the past. Downers Grove, IL: InterVarsity Press.

McCullough, M. E., \& Worthington, Jr., J. L. (1994). Encouraging clients to forgive people who have hurt them: Review, critique, and research prospectus. Journal of Psychology and Theology, 22, 3-20.

McEwen, B. S. (1998). Protective and damaging effects of stress mediators. New England Journal of Medicine, 338, 171-179.

McEwen, B. S., \& Stellar, E. (1993). Stress and the individual: Mechanisms leading to disease. Archives of Internal Medicine, 153, 2093-2101.

Miller, T. Q., Smith, T. W., Turner, C. W., Guijarro, M. L., \& Hallet, A. J. (1996). Meta-analytic review of research on hostility and physical health. Psychological Bulletin, 119, 322-348.

Schwenkmezger, P., \& Hank, P. (1996). Anger expression and blood pressure. In C. D. Spielberger, and I. G. Sarason (Eds.). Stress and emotion: Anxiety, anger, and curiosity, Vol. 16 (241-259). Washington D. C.: Taylor \& Francis.

Tennen, H., \& Affleck, G. (1990, Sep.). Blaming others for threatening events. Psychological Bulletin, 108, 209-232.

Thoresen, C. E., Harris, A. H. S., \& Luskin, F. (1999). Forgiveness and health: An unanswered question. In M. E. McCullough, K. I. Pargament \& C. E. Thoresen (Eds.) Forgiveness: Theory, research, and practice (pp. 254-280). New York: Guilford Press.

Thoresen, C. E., Luskin, F., \& Harris, A. H. S. (1998). Science and forgiveness interventions: Reflections and recommendations. In E. L. Worthington, Jr. (Ed.) Dimensions of forgiveness (pp. 163-190). Philadelphia, PA: Templeton Foundation Press.

Vrana, S. R., \& Lang, P. J. (1990). Fear imagery and the startle-probe reflex. Journal of Abnormal Psychology, 99, 189-197.

Wenneberg, S. R., Schneider, R. H., Walton, K. G., MacLean, C. R. K., et al. (1997). Anger expression correlates with platelet aggregation. Behavioral Medicine, 22, 174-177. 
Williams, R., \& Williams, V. (1993). Anger kills: Seventeen strategies for controlling the hostility that can harm your health. New York: Harper Perennial.

Witvliet, C.V.O. (1997). Traumatic Intrusive Imagery As An Emotional Memory Phenomenon: A Review of research and explanatory information processing theories. Clinical Psychology Review, 17, 509-536.

Witvliet, C. V. O., \& Vrana, S. R. (1995). Psychophysiological responses as indices of affective dimensions. Psychophysiology, 32, 436-443.

Witvliet, C. V. O., \& Vrana, S. R. (2000). Emotional imagery, the visual startle, and covariation bias: An affective matching account. Biological Psychology, 52, 187-204.

Worthington, E. L. Jr. (1998). Empirical research in forgiveness: Looking backward, looking forward. In E. L. Worthington, Jr. (Ed.) Dimensions of forgiveness (pp. 321-339). Philadelphia, PA: Templeton Foundation Press.

Yogo, Y., Hama, H., Yogo, M, \& Matsuyama, Y. (1995). A study of physiological response during emotional imaging. Perceptual and Motor Skills, 81, 43-49. 


\section{Footnotes}

${ }^{1}$ EMG increases at the orbicularis oculi (under the eye) also were significantly greater during unforgiving imagery, but zygomatic (cheek) EMG showed no effects.

${ }^{2}$ Diastolic blood pressure was significantly higher throughout unforgiving imagery; systolic blood pressure was significantly greater during unforgiving imagery epochs two and three.

${ }^{3}$ Further analyses supported this theoretical rationale. Physiology did not differ between the hurt and grudge conditions, nor between the empathy and forgiveness conditions, but physiology did differ significantly for each of the two unforgiving conditions compared to each of the two forgiving conditions (all heart rate, skin conductance, MAP, and corrugator Fs $>4$, ps $<.05$; except MAP differences between grudge and both empathy and forgiveness conditions were marginal: $\underline{\text { Fs }}>3.1$, s $\leq .081)$.

${ }^{4}$ In the interest of space, we do not report epoch effects. Yet, the figures depict data across epochs to assist readers in understanding the physiological results across imagery and recovery periods.

${ }^{5}$ Individual difference variables (e.g., sex, offense severity, whether the offender had apologized, whether the offender and victim had repaired their relationship, and the degree to which the victim had held a grudge, had desired revenge against, had empathized with, or had forgiven the offender) did not have significant effects on heart rate, mean arterial pressure, skin conductance, and corrugator EMG. 
Table 1

Means and (Standard Deviations) of Self Report Data for the Unforgiving (Hurt and Grudge) and Forgiving

(Empathy and Forgiveness) Imagery Conditions

\section{Imagery Conditions}

Measure Unforgiving Forgiving

$\begin{array}{lcc}\text { Valence } & 5.63 & 13.21 \\ \text { Arousal } & (2.72) & (3.27) \\ & 15.34 & 7.21 \\ \text { Control } & (2.95) & (3.68) \\ & (3.37 & 13.03 \\ \text { Sadness } & 11.71 & (3.43) \\ & (4.41) & 7.14 \\ \text { Anger } & 15.75 & (4.28) \\ & (2.63) & 5.11 \\ \text { Empathy } & 3.87 & (3.84) \\ & (3.35) & 13.91 \\ \text { Forgiveness } & 4.08 & (3.55) \\ & (3.27) & 14.64 \\ & & (3.92)\end{array}$

Note. Participants' ratings about how they felt during each type of imagery were converted to a 0 - 20 scale. For valence, 0 is strongly negative, and 20 is strongly positive. For arousal and control, 0 is very low, and 20 is very high. For sadness, anger, empathy, and forgiveness, 0 means "not at all,” and 20 means "completely.” 


\section{Figure Captions}

Figure 1. Corrugator EMG change scores from baseline during the 16-second imagery and 8-second recovery periods.

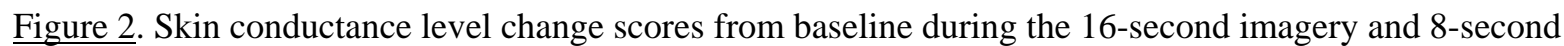
recovery periods.

Figure 3. Heart rate change scores from baseline during the 16-second imagery and 8-second recovery periods. Figure 4. Mean arterial pressure change scores from baseline during the 16-second imagery and 8-second recovery periods. 


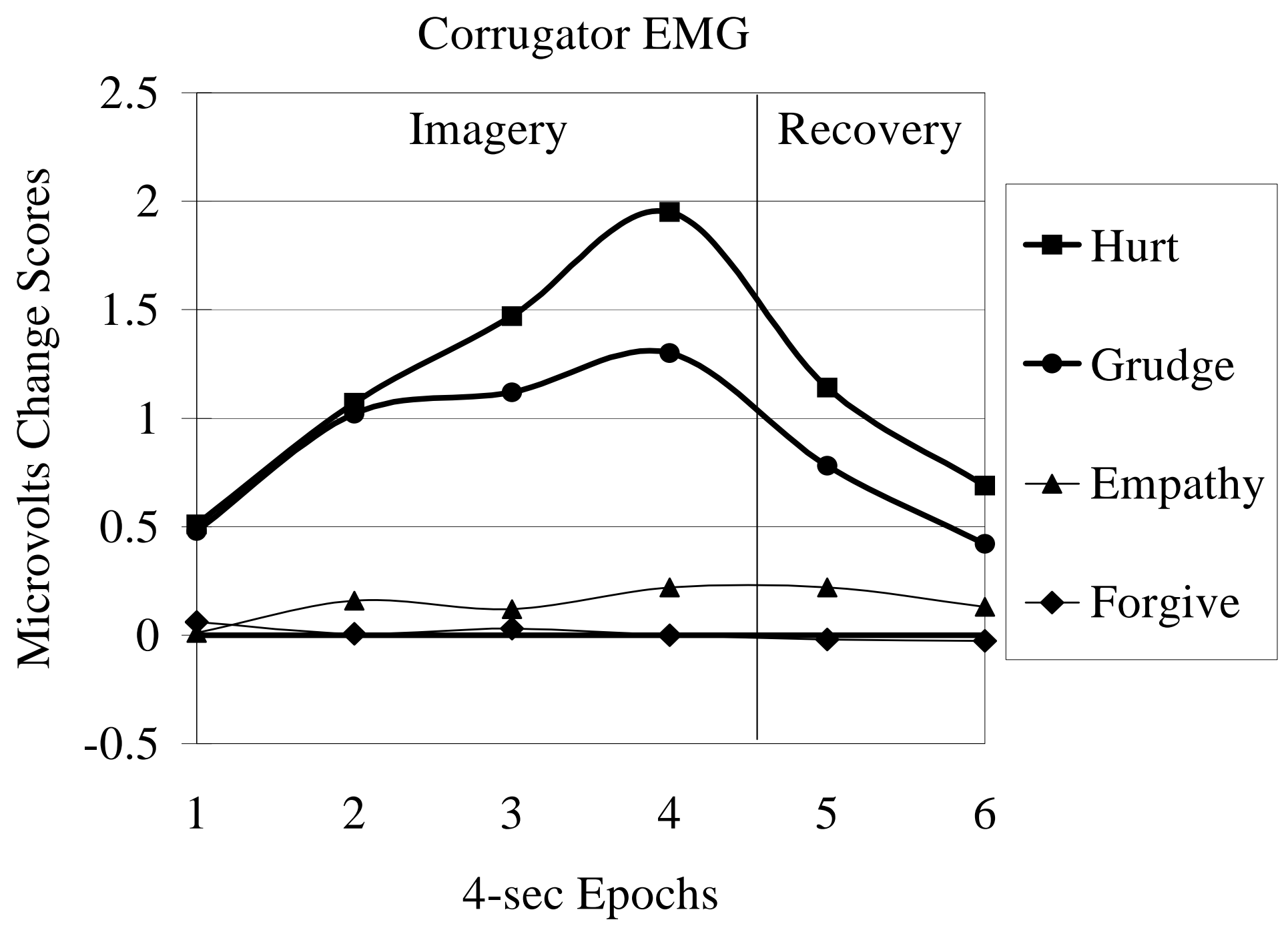




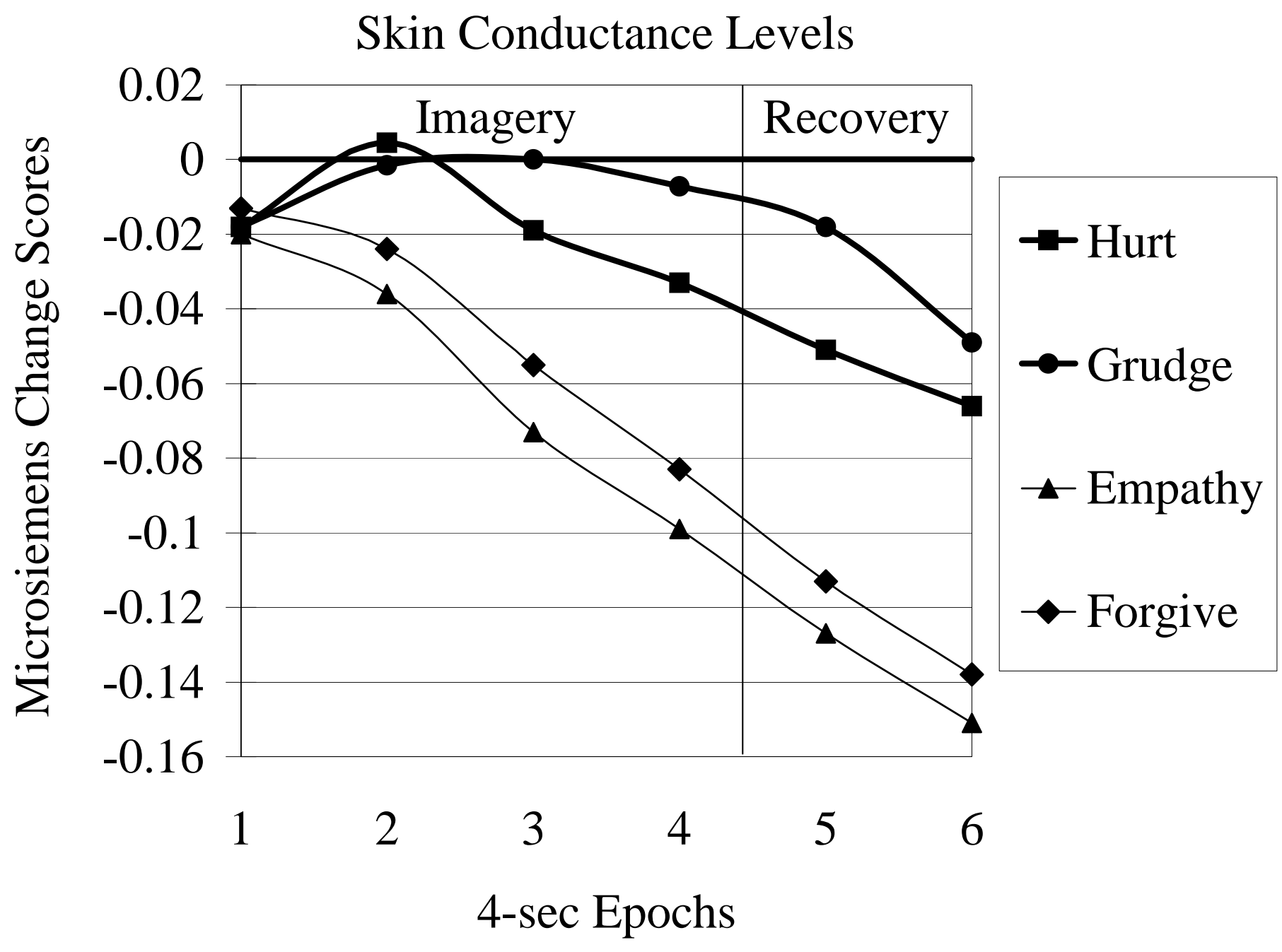




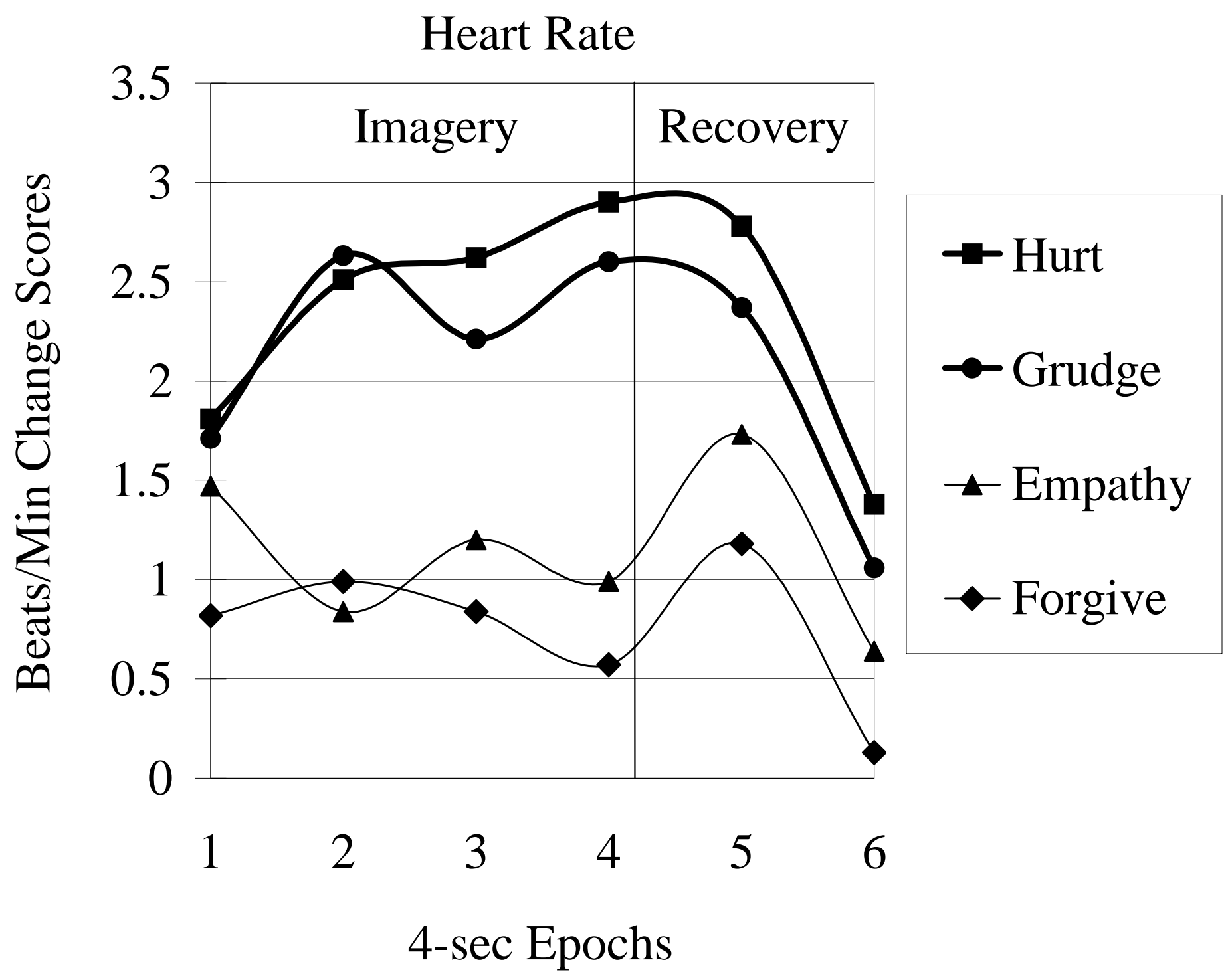




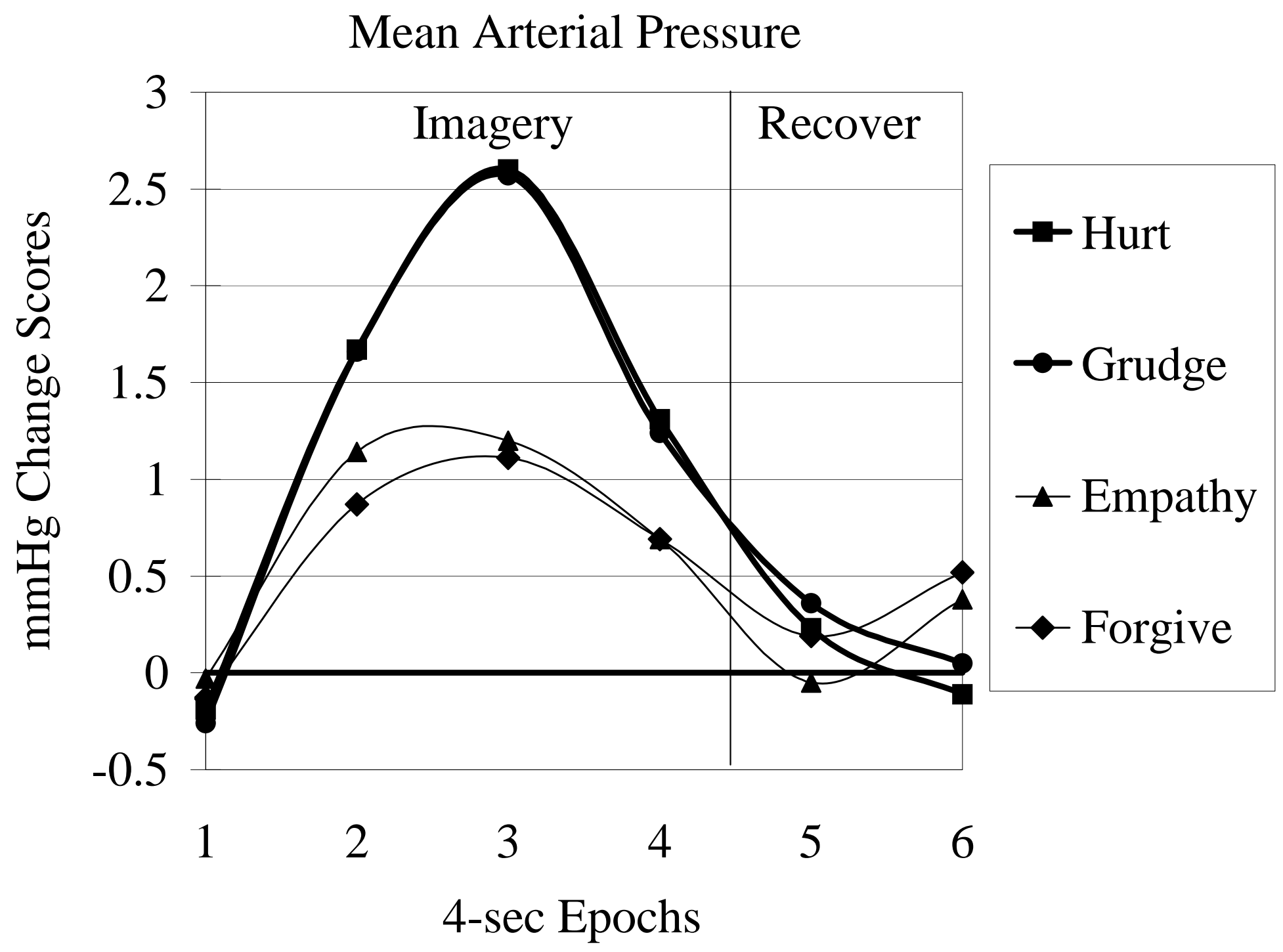


